\title{
Determinants of the RFMLR Circulant Matrices with Perrin, Padovan, Tribonacci, and the Generalized Lucas Numbers
}

\author{
Zhaolin Jiang, ${ }^{1}$ Nuo Shen, ${ }^{1,2}$ and Juan $\mathrm{Li}^{1,2}$ \\ ${ }^{1}$ Department of Mathematics, Linyi University, Linyi, Shandong 276000, China \\ ${ }^{2}$ Department of Mathematics, Shandong Normal University, Jinan, Shandong 250000, China
}

Correspondence should be addressed to Nuo Shen; shennuo2007@aliyun.com

Received 22 July 2013; Revised 4 December 2013; Accepted 6 December 2013; Published 29 January 2014

Academic Editor: George Jaiani

Copyright (C) 2014 Zhaolin Jiang et al. This is an open access article distributed under the Creative Commons Attribution License, which permits unrestricted use, distribution, and reproduction in any medium, provided the original work is properly cited.

\begin{abstract}
The row first-minus-last right (RFMLR) circulant matrix and row last-minus-first left (RLMFL) circulant matrices are two special pattern matrices. By using the inverse factorization of polynomial, we give the exact formulae of determinants of the two pattern matrices involving Perrin, Padovan, Tribonacci, and the generalized Lucas sequences in terms of finite many terms of these sequences.
\end{abstract}

\section{Introduction}

Several special matrices arise frequently in many fields including image processing, communications, medicine, and signal encoding [1]. The application of a block-circulant matrix approach for singular value decomposition rendered the analysis independent of tracer arrival time to improve the results in [2]. Yin et al. introduced fast algorithms for reconstructing signals from incomplete Toeplitz and circulant measurements and showed that Toeplitz and circulant matrices not only were as effective as random matrices for signal encoding but also permitted much faster decoding in [3]. Wu et al. proposed a technique that was made time-shift insensitive by the use of a block-circulant matrix for deconvolution with (oSVD) and without (cSVD) minimization of oscillation of the derived residue function in [4].

The circulant matrices [5, 6], a fruitful subject of research, have in recent years been extended in many directions. The $f(x)$-circulant matrices are another natural extension of this well-studied class and can be found in [7-10]. The $f(x)$ circulant matrix has a wide application, especially on the generalized cyclic codes [7]. The properties and structures of the $x^{n}+x-1$-circulant matrices, which are called the row first-minus-last right (RFMLR) circulant matrices, are better than those of the general $f(x)$-circulant matrices, so it is significant that we give our attention to them.
We first introduce the definitions of the row first-minuslast right (RFMLR) circulant matrices and row last-minusfirst left (RLMFL) circulant matrices. As regards their more properties, please refer to $[11,12]$.

Definition 1. A row first-minus-last right (RFMLR) circulant matrix with the first row $\left(a_{1}, a_{2}, \ldots, a_{n}\right)$, denoted by $\operatorname{RFMLRcircfr}\left(a_{1}, a_{2}, \ldots, a_{n}\right)$, is meant to be a square matrix of the form

$$
\mathbf{A}=\left(\begin{array}{cccc}
a_{1} & a_{2} & \ldots & a_{n} \\
a_{n} & a_{1}-a_{n} & \ldots & a_{n-1} \\
\vdots & \vdots & \ddots & \vdots \\
a_{3} & a_{4}-a_{3} & \ldots & a_{2} \\
a_{2} & a_{3}-a_{2} & \ldots & a_{1}-a_{n}
\end{array}\right) .
$$

We define matrix $\Theta_{(1,-1)}$ as the basic RFMLR circulant matrix; that is,

$$
\begin{aligned}
\Theta_{(1,-1)} & =\left(\begin{array}{ccccc}
0 & 1 & 0 & \cdots & 0 \\
\vdots & \ddots & \ddots & \ddots & \vdots \\
\vdots & \ddots & \ddots & \ddots & 0 \\
0 & \cdots & \cdots & 0 & 1 \\
1 & -1 & 0 & \cdots & 0
\end{array}\right) \\
& =\operatorname{RFMLRcircfr}(0,1,0, \ldots, 0) .
\end{aligned}
$$


Definition 2. A row last-minus-first left (RLMFL) circulant matrix with the first row $\left(a_{1}, a_{2}, \ldots, a_{n}\right)$, denoted by $\operatorname{RLMFLcircfr}\left(a_{1}, a_{2}, \ldots, a_{n}\right)$, is meant to be a square matrix of the form

$$
\mathbf{B}=\left(\begin{array}{cccc}
a_{1} & \cdots & a_{n-1} & a_{n} \\
a_{2} & \cdots & a_{n}-a_{1} & a_{1} \\
\vdots & \therefore & \vdots & \vdots \\
a_{n-1} & \cdots & a_{n-3}-a_{n-2} & a_{n-2} \\
a_{n}-a_{1} & \cdots & a_{n-2}-a_{n-1} & a_{n-1}
\end{array}\right) .
$$

Let $\mathbf{A}=\operatorname{RFMLRcircfr}\left(a_{n}, a_{n-1}, \ldots, a_{1}\right)$ and $\mathbf{B}=$ RLMFLcircfr $\left(a_{1}, a_{2}, \ldots, a_{n}\right)$. By explicit computation, we find

$$
\mathbf{B}=\mathbf{A} \widehat{\mathbf{I}},
$$

where $\widehat{\mathbf{I}}$ is the backward identity matrix of the form

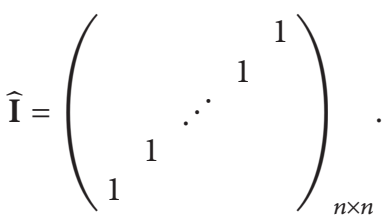

There are many interests in properties and generalization of some special matrices with famous numbers. Jaiswal evaluated some determinants of circulant whose elements are the generalized Fibonacci numbers [13]. Lin gave the determinant of the Fibonacci-Lucas quasi-cyclic matrices [14]. Lind presented the determinants of circulant and skew circulant involving Fibonacci numbers in [15]. Shen et al. [16] discussed the determinant of circulant matrix involving Fibonacci and Lucas numbers. Akbulak and Bozkurt [17] gave the norms of Toeplitz involving Fibonacci and Lucas numbers.

The determinant problems of the row first-minus-last right (RFMLR) circulant matrices and row last-minusfirst left (RLMFL) circulant matrices involving the Perrin, Padovan, Tribonacci, and the generalized Lucas sequences are considered in this paper. The exact formulae of determinants are presented by using some terms of these sequences. The techniques used herein are based on the inverse factorization of polynomial.

The Perrin and Padovan sequences $\left\{R_{n}\right\}$ and $\left\{\mathbb{P}_{n}\right\}[18-20]$ are defined by a third-order recurrence:

$$
\begin{array}{ll}
R_{n}=R_{n-2}+R_{n-3}, & n \geq 3, \\
\mathbb{P}_{n}=\mathbb{P}_{n-2}+\mathbb{P}_{n-3}, \quad n \geq 3,
\end{array}
$$

with the initial conditions $R_{0}=3, R_{1}=0$, and $R_{2}=2$, and $\mathbb{P}_{0}=1, \mathbb{P}_{1}=1$, and $\mathbb{P}_{2}=1$.

The Tribonacci and the generalized Lucas sequences $\left\{T_{n}\right\}$ and $\left\{\mathbb{L}_{n}\right\}[20,21]$ are defined by a third-order recurrence:

$$
\begin{array}{ll}
T_{n}=T_{n-1}+T_{n-2}+T_{n-3}, & n \geq 3, \\
\mathbb{L}_{n}=\mathbb{L}_{n-1}+\mathbb{L}_{n-2}+\mathbb{L}_{n-3}, & n \geq 3,
\end{array}
$$

with the initial conditions $T_{0}=0, T_{1}=1$, and $T_{2}=1$ and $\mathbb{L}_{0}=3, \mathbb{L}_{1}=1$, and $\mathbb{L}_{2}=3$.
The first few members of these sequences are given as follows:

$\begin{array}{cccccccc}n & 0 & 1 & 2 & 3 & 4 & 5 & 6 \\ R_{n} & 3 & 0 & 2 & 3 & 2 & 5 & 5 \\ \mathbb{P}_{n} & 1 & 1 & 1 & 2 & 2 & 3 & 4 \\ T_{n} & 0 & 1 & 1 & 2 & 4 & 7 & 13 \\ \mathbb{Z}_{n} & 3 & 1 & 3 & 7 & 11 & 21 & 39 .\end{array}$

Recurrences (6) and (7) involve the characteristic equation $x^{3}-x-1=0$. If its roots are denoted by $r_{1}, r_{2}, r_{3}$, then the following equalities can be derived:

$$
\begin{gathered}
r_{1}+r_{2}+r_{3}=0, \\
r_{1} r_{2}+r_{1} r_{3}+r_{2} r_{3}=-1, \\
r_{1} r_{2} r_{3}=1 .
\end{gathered}
$$

Moreover, the Binet form for the Perrin sequence is

$$
R_{n}=r_{1}^{n}+r_{2}^{n}+r_{3}^{n}
$$

and the Binet form for Padovan sequence is

$$
\mathbb{P}_{n}=a_{1} r_{1}^{n}+a_{2} r_{2}^{n}+a_{3} r_{3}^{n},
$$

where

$$
a_{i}=\prod_{\substack{j=1 \\ j \neq i}}^{3} \frac{r_{j}-1}{r_{i}-r_{j}}, \quad i=1,2,3 .
$$

Recurrences (8) as well imply the characteristic equation $x^{3}-$ $x^{2}-x-1=0$. If its roots are denoted by $t_{1}, t_{2}, t_{3}$, then we have

$$
\begin{gathered}
t_{1}+t_{2}+t_{3}=1, \\
t_{1} t_{2}+t_{1} t_{3}+t_{2} t_{3}=-1, \\
t_{1} t_{2} t_{3}=1 .
\end{gathered}
$$

Furthermore, an exact expression for the $n$th Tribonacci number can be given explicitly by

$$
\begin{aligned}
T_{n}= & \frac{t_{1}^{n+1}}{\left(t_{1}-t_{2}\right)\left(t_{1}-t_{3}\right)}+\frac{t_{2}^{n+1}}{\left(t_{2}-t_{1}\right)\left(t_{2}-t_{3}\right)} \\
& +\frac{t_{3}^{n+1}}{\left(t_{3}-t_{1}\right)\left(t_{3}-t_{2}\right)} \\
= & \frac{t_{1}^{n}}{-t_{1}^{2}+4 t_{1}-1}+\frac{t_{2}^{n}}{-t_{2}^{2}+4 t_{2}-1}+\frac{t_{3}^{n}}{-t_{3}^{2}+4 t_{3}-1} .
\end{aligned}
$$

This can be written in a slightly more concise form (the Binet form) as

$$
T_{n}=b_{1} t_{1}^{n}+b_{2} t_{2}^{n}+b_{3} t_{3}^{n}
$$

where $b_{i}$ is the $i$ th root of the polynomial $44 y^{3}-2 y-1$. And the Binet form for the generalized Lucas sequence is

$$
\mathbb{L}_{n}=t_{1}^{n}+t_{2}^{n}+t_{3}^{n} .
$$




\section{Main Results}

By Proposition 5.1 in [7] and properties of RFMLR circulant matrices [12], we deduce the following lemma.

Lemma 3. Let $\mathbf{A}=\operatorname{RFMLR\operatorname {circf}r}\left(a_{1}, a_{2}, \ldots, a_{n}\right)$ and $\varepsilon_{i}(i=$ $1,2, \ldots, n)$ be the roots of the characteristic equation of $\boldsymbol{\Theta}_{(1,-1)}$. Then the eigenvalues of $\mathbf{A}$ are given by

$$
\lambda_{i}=f\left(\varepsilon_{i}\right)=\sum_{j=1}^{n} a_{j} \varepsilon_{i}^{j-1}, \quad i=1,2, \ldots, n,
$$

and the determinant of $\mathbf{A}$ is given by

$$
\operatorname{det} \mathbf{A}=\prod_{i=1}^{n} \lambda_{i}=\prod_{i=1}^{n} \sum_{j=1}^{n} a_{j} \varepsilon_{i}^{j-1}
$$

Lemma 4. Suppose $\varepsilon_{i}(i=1,2, \ldots, n)$ are the roots of the characteristic equation of $\boldsymbol{\Theta}_{(1,-1)}$. If $a=0$, then

$$
\begin{aligned}
\prod_{i=1}^{n}\left(a \varepsilon_{i}^{3}+b \varepsilon_{i}^{2}+c \varepsilon_{i}+d\right)= & \prod_{i=1}^{n}\left(b \varepsilon_{i}^{2}+c \varepsilon_{i}+d\right) \\
= & d^{n}+b^{n-1}(b+c+d) \\
& +d\left(\delta_{1}^{n-1}+\delta_{2}^{n-1}\right)-\left(\delta_{1}^{n}+\delta_{2}^{n}\right),
\end{aligned}
$$

where $a, b, c \in \mathbb{R}$ and

$$
\begin{aligned}
& \delta_{1}=\frac{-c+\sqrt{c^{2}-4 b d}}{2}, \\
& \delta_{2}=\frac{-c-\sqrt{c^{2}-4 b d}}{2} .
\end{aligned}
$$

If $a \neq 0$, then

$$
\begin{aligned}
\prod_{i=1}^{n}\left(a \varepsilon_{i}^{3}+b \varepsilon_{i}^{2}+c \varepsilon_{i}+d\right) \\
=\frac{(-a)^{n}}{2}\left(-\Delta_{n}^{2}+\Delta_{2 n}+2 \Delta_{n+1}+2 \Delta_{n}\right) \\
\quad+\frac{(-a)^{n-1}}{2} d\left(\Delta_{n-1}^{2}-\Delta_{2(n-1)}+2 \Delta_{n-1}\right) \\
\quad+(-a)^{n-1}\left(-b \Delta_{n}+a+b+c+d\right)+d^{n},
\end{aligned}
$$

where $\Delta_{n}=\delta_{1}^{n}+\delta_{2}^{n}+\delta_{3}^{n}$, and $\delta_{1}, \delta_{2}, \delta_{3}$ are the roots of the equation $a x^{3}+b x^{2}+c x+d=0$.

Proof. Since $\varepsilon_{i}(i=1,2, \ldots, n)$ are the roots of the characteristic equation of $\boldsymbol{\Theta}_{(1,-1)}, g(x)=x^{n}+x-1$ can be factored as

$$
x^{n}+x-1=\prod_{i=1}^{n}\left(x-\varepsilon_{i}\right) .
$$

Let $\delta_{1}, \delta_{2}, \delta_{3}$ be the roots of the equation $a x^{3}+b x^{2}+c x+d=0$.
If $a=0$, please see [12] for details of the proof.

If $a \neq 0$, then

$$
\begin{aligned}
& \prod_{i=1}^{n}\left(a \varepsilon_{i}^{3}+b \varepsilon_{i}^{2}+c \varepsilon_{i}+d\right) \\
&=a^{n} \prod_{i=1}^{n}\left(\varepsilon_{i}^{3}+\frac{b}{a} \varepsilon_{i}^{2}+\frac{c}{a} \varepsilon_{i}+\frac{d}{a}\right) \\
&=a^{n} \prod_{i=1}^{n}\left(\varepsilon_{i}-\delta_{1}\right)\left(\varepsilon_{i}-\delta_{2}\right)\left(\varepsilon_{i}-\delta_{3}\right) \\
&=(-a)^{n} \prod_{i=1}^{n}\left(\delta_{1}-\varepsilon_{i}\right) \prod_{i=1}^{n}\left(\delta_{2}-\varepsilon_{i}\right) \prod_{i=1}^{n}\left(\delta_{3}-\varepsilon_{i}\right) \\
&=(-a)^{n}\left(\delta_{1}^{n}+\delta_{1}-1\right)\left(\delta_{2}^{n}+\delta_{2}-1\right)\left(\delta_{3}^{n}+\delta_{3}-1\right) \\
&=(-a)^{n}\left\{\left(\delta_{1} \delta_{2} \delta_{3}\right)^{n}+\delta_{1} \delta_{2} \delta_{3}\left[\left(\delta_{1} \delta_{2}\right)^{n-1}+\left(\delta_{1} \delta_{3}\right)^{n-1}\right.\right. \\
&\left.+\left(\delta_{2} \delta_{3}\right)^{n-1}\right] \\
&-\left[\left(\delta_{1} \delta_{2}\right)^{n}+\left(\delta_{1} \delta_{3}\right)^{n}+\left(\delta_{2} \delta_{3}\right)^{n}\right] \\
&+\delta_{1} \delta_{2} \delta_{3}\left(\delta_{1}^{n-1}+\delta_{2}^{n-1}+\delta_{3}^{n-1}\right) \\
&-\left[\delta_{1}^{n}\left(\delta_{2}+\delta_{3}\right)+\delta_{2}^{n}\left(\delta_{1}+\delta_{3}\right)\right. \\
&\left.\quad+\delta_{3}^{n}\left(\delta_{1}+\delta_{2}\right)\right]+\left(\delta_{1}^{n}+\delta_{2}^{n}+\delta_{3}^{n}\right) \\
&+ \delta_{1} \delta_{2} \delta_{3}-\left(\delta_{1} \delta_{2}+\delta_{1} \delta_{3}+\delta_{2} \delta_{3}\right) \\
&+\left.\left(\delta_{1}+\delta_{2}+\delta_{3}\right)-1\right\} .
\end{aligned}
$$

Let $\Delta_{n}=\delta_{1}^{n}+\delta_{2}^{n}+\delta_{3}^{n}$. We derive $\left(\delta_{1} \delta_{2}\right)^{n}+\left(\delta_{1} \delta_{3}\right)^{n}+\left(\delta_{2} \delta_{3}\right)^{n}=$ $\left(\Delta_{n}^{2}-\Delta_{2 n}\right) / 2$ from $\left(\delta_{1}^{n}+\delta_{2}^{n}+\delta_{3}^{n}\right)^{2}=\delta_{1}^{2 n}+\delta_{2}^{2 n}+\delta_{3}^{2 n}+2\left[\left(\delta_{1} \delta_{2}\right)^{n}+\right.$ $\left.\left(\delta_{1} \delta_{3}\right)^{n}+\left(\delta_{2} \delta_{3}\right)^{n}\right]$. Taking the relation of roots and coefficients

$$
\begin{aligned}
\delta_{1}+\delta_{2}+\delta_{3} & =-\frac{b}{a} \\
\delta_{1} \delta_{2}+\delta_{1} \delta_{3}+\delta_{2} \delta_{3} & =\frac{c}{a} \\
\delta_{1} \delta_{2} \delta_{3} & =-\frac{d}{a}
\end{aligned}
$$

into account, we deduce that

$$
\begin{aligned}
\prod_{i=1}^{n}\left(a \varepsilon_{i}^{3}+b \varepsilon_{i}^{2}+c \varepsilon_{i}+d\right) \\
=\frac{(-a)^{n}}{2}\left(-\Delta_{n}^{2}+\Delta_{2 n}+2 \Delta_{n+1}+2 \Delta_{n}\right) \\
\quad+\frac{(-a)^{n-1}}{2} d\left(\Delta_{n-1}^{2}-\Delta_{2(n-1)}+2 \Delta_{n-1}\right) \\
\quad+(-a)^{n-1}\left(-b \Delta_{n}+a+b+c+d\right)+d^{n} .
\end{aligned}
$$

The proof is completed. 
We present the exact formulae of determinants of the RFMLR and RLMFL circulant matrices involving four kinds of famous numbers and the detailed process.

\subsection{Determinants of the RFMLR and RLMFL Circulant Matrix} Involving Perrin Sequence

Theorem 5. Let $\mathbf{C}=R F M L R \operatorname{circfr}\left(R_{1}, R_{2}, \ldots, R_{n}\right)$. If $n$ is odd, then

$$
\operatorname{det} \mathbf{C}=\frac{\left(-x_{1}+x_{2}+x_{3}\right)}{\mathscr{Y}_{1}}
$$

and if $n$ is even, then

$$
\operatorname{det} \mathbf{C}=\frac{\left(x_{1}-x_{2}-x_{3}\right)}{\mathscr{Y}_{1}} \text {, }
$$

where

$$
\begin{gathered}
\mathscr{X}_{1}=R_{n}^{n}\left(X_{n}^{2}-X_{2 n}-2 X_{n+1}\right), \\
\mathscr{X}_{2}=R_{n+1} R_{n}^{n-1}\left(X_{n-1}^{2}-X_{2(n-1)}+2 X_{n-1}\right), \\
X_{3}=2 R_{n}^{n-1}\left(R_{n+2} X_{n}+3 X_{n}-5\right)+2 R_{n+1}^{n}, \\
\mathscr{Y}_{1}=Y_{n}^{2}-Y_{n-1}^{2}-Y_{2 n}+Y_{2(n-1)} \\
-2 Y_{n+1}-4 Y_{n}-2 Y_{n-1}, \\
X_{n}=x_{1}^{n}+x_{2}^{n}+x_{3}^{n}, \\
Y_{n}=y_{1}^{n}+y_{2}^{n}+y_{3}^{n},
\end{gathered}
$$

where $x_{1}, x_{2}, x_{3}$ and $y_{1}, y_{2}, y_{3}$ are the roots of the equation $R_{n} x^{3}+\left(R_{n-1}+3\right) x^{2}-\left(R_{n-3}-2\right) x-R_{n+1}=0, x^{3}+x^{2}-1=0$, respectively.

Proof. Obviously, $\mathrm{C}$ has the form

$$
\mathbf{C}=\left(\begin{array}{cccc}
R_{1} & R_{2} & \ldots & R_{n} \\
R_{n} & R_{1}-R_{n} & \ldots & R_{n-1} \\
\vdots & \vdots & \ddots & \vdots \\
R_{3} & R_{4}-R_{3} & \ldots & R_{2} \\
R_{2} & R_{3}-R_{2} & \ldots & R_{1}-R_{n}
\end{array}\right)
$$

In the light of Lemma 3 and the Binet form (11) and (10), we have

$$
\begin{aligned}
\operatorname{det} \mathbf{C} & =\prod_{i=1}^{n}\left(R_{1}+R_{2} \varepsilon_{i}+\cdots+R_{n} \varepsilon_{i}^{n-1}\right) \\
& =\prod_{i=1}^{n} \sum_{k=1}^{n} \sum_{j=1}^{3} r_{j}^{k} \varepsilon_{i}^{k-1} \\
& =\prod_{i=1}^{n} \sum_{j=1}^{3} \frac{r_{j}\left(1-r_{j}^{n} \varepsilon_{i}^{n}\right)}{1-r_{j} \varepsilon_{i}}
\end{aligned}
$$

$$
\begin{aligned}
= & \prod_{i=1}^{n} \frac{R_{n} \varepsilon_{i}^{3}+\left(R_{n-1}+3\right) \varepsilon_{i}^{2}}{-\varepsilon_{i}^{3}-\varepsilon_{i}^{2}+1} \\
& -\frac{\left(R_{n-3}-2\right) \varepsilon_{i}+R_{n+1}}{-\varepsilon_{i}^{3}-\varepsilon_{i}^{2}+1} .
\end{aligned}
$$

By Lemma 4 and recurrence (6), we obtain

$$
\begin{aligned}
\prod_{i=1}^{n}[ & \left.R_{n} \varepsilon_{i}^{3}+\left(R_{n-1}+3\right) \varepsilon_{i}^{2}-\left(R_{n-3}-2\right) \varepsilon_{i}-R_{n+1}\right] \\
=\left(-R_{n}\right)^{n-1} & {\left[R_{n} X_{n}^{2}-R_{n+1} X_{n-1}^{2}-R_{n} X_{2 n}\right.} \\
& +R_{n+1} X_{2(n-1)}-2 R_{n} X_{n+1} \\
& \left.-2\left(R_{n+2}+3\right) X_{n}-2 R_{n+1} X_{n-1}+10\right] \times 2^{-1} \\
+\left(-R_{n+1}\right)^{n} &
\end{aligned}
$$

where $X_{n}=x_{1}^{n}+x_{2}^{n}+x_{3}^{n}$ and $x_{1}, x_{2}, x_{3}$ are the roots of the equation $R_{n} x^{3}+\left(R_{n-1}+3\right) x^{2}-\left(R_{n-3}-2\right) x-R_{n+1}=0$. And

$$
\begin{aligned}
\prod_{i=1}^{n}\left(-\varepsilon_{i}^{3}-\varepsilon_{i}^{2}+1\right)= & \frac{1}{2}\left(-Y_{n}^{2}+Y_{n-1}^{2}+Y_{2 n}-Y_{2(n-1)}\right) \\
& +Y_{n+1}+2 Y_{n}+Y_{n-1},
\end{aligned}
$$

where $Y_{n}=y_{1}^{n}+y_{2}^{n}+y_{3}^{n}$ and $y_{1}, y_{2}, y_{3}$ are the roots of the equation $x^{3}+x^{2}-1=0$. Consequently, if $n$ is odd, then

$$
\operatorname{det} \mathbf{C}=\frac{\left(-x_{1}+x_{2}+x_{3}\right)}{\mathscr{Y}_{1}},
$$

and if $n$ is even, then

$$
\operatorname{det} \mathbf{C}=\frac{\left(x_{1}-X_{2}-x_{3}\right)}{\mathscr{Y}_{1}},
$$

where

$$
\begin{aligned}
\mathscr{X}_{1}= & R_{n}^{n}\left(X_{n}^{2}-X_{2 n}-2 X_{n+1}\right), \\
\mathscr{X}_{2}= & R_{n+1} R_{n}^{n-1}\left(X_{n-1}^{2}-X_{2(n-1)}+2 X_{n-1}\right), \\
\mathscr{X}_{3}= & 2 R_{n}^{n-1}\left(R_{n+2} X_{n}+3 X_{n}-5\right)+2 R_{n+1}^{n}, \\
\mathscr{Y}_{1}= & Y_{n}^{2}-Y_{n-1}^{2}-Y_{2 n}+Y_{2(n-1)} \\
& -2 Y_{n+1}-4 Y_{n}-2 Y_{n-1} .
\end{aligned}
$$

The proof is completed.

Theorem 6. Let $\mathbf{D}=R F M L R \operatorname{circfr}\left(R_{n}, \ldots, R_{1}\right)$. Then

$$
\operatorname{det} \mathbf{D}=\frac{2\left[\alpha_{4}+\left(R_{n}-3\right)\left(\alpha_{1}^{n-1}+\alpha_{2}^{n-1}\right)-\left(\alpha_{1}^{n}+\alpha_{2}^{n}\right)\right]}{-R_{n+2} R_{n-5}+R_{2 n-3}+2 R_{n+4}+4},
$$


where

$$
\begin{aligned}
& \alpha_{1}=\frac{\left(-R_{n+2}-1+\sqrt{\alpha_{3}}\right)}{2}, \\
& \alpha_{2}=\frac{\left(-R_{n+2}-1-\sqrt{\alpha_{3}}\right)}{2}, \\
& \alpha_{3}=\left(R_{n+2}+1\right)^{2}-4\left(R_{n+1}+2\right)\left(R_{n}-3\right), \\
& \alpha_{4}=\left(R_{n}-3\right)^{n}+R_{n+5}\left(R_{n+1}+2\right)^{n-1} .
\end{aligned}
$$

Proof. The matrix $\mathbf{D}$ has the form

$$
\mathbf{D}=\left(\begin{array}{cccc}
R_{n} & R_{n-1} & \ldots & R_{1} \\
R_{1} & R_{n}-R_{1} & \ldots & R_{2} \\
\vdots & \vdots & \ddots & \vdots \\
R_{n-2} & R_{n-3}-R_{n-2} & \ldots & R_{n-1} \\
R_{n-1} & R_{n-2}-R_{n-1} & \ldots & R_{n}-R_{n-1}
\end{array}\right)
$$

According to Lemma 3 and the Binet form (11) and (10), we have

$$
\begin{aligned}
\operatorname{det} \mathbf{D} & =\prod_{i=1}^{n}\left(R_{n}+R_{n-1} \varepsilon_{i}+\cdots+R_{1} \varepsilon_{i}^{n-1}\right) \\
& =\prod_{i=1}^{n} \sum_{k=0}^{n-1} \sum_{j=1}^{3} r_{j}^{n-k} \varepsilon_{i}^{k} \\
& =\prod_{i=1}^{n} \sum_{j=1}^{3} \frac{r_{j}^{n+1}-r_{j} \varepsilon_{i}^{n}}{r_{j}-\varepsilon_{i}} \\
& =\prod_{i=1}^{n} \frac{\left(R_{n+1}+2\right) \varepsilon_{i}^{2}+\left(R_{n+2}+1\right) \varepsilon_{i}+R_{n}-3}{-\varepsilon_{i}^{3}+\varepsilon_{i}+1} .
\end{aligned}
$$

Using Lemma 4 and recurrence (6), we obtain

$$
\begin{aligned}
\prod_{i=1}^{n} & {\left[\left(R_{n+1}+2\right) \varepsilon_{i}^{2}+\left(R_{n+2}+1\right) \varepsilon_{i}+R_{n}-3\right] } \\
& =\alpha_{4}+\left(R_{n}-3\right)\left(\alpha_{1}^{n-1}+\alpha_{2}^{n-1}\right)-\left(\alpha_{1}^{n}+\alpha_{2}^{n}\right),
\end{aligned}
$$

where

$$
\begin{aligned}
\alpha_{1} & =\frac{\left(-R_{n+2}-1+\sqrt{\alpha_{3}}\right)}{2}, \\
\alpha_{2} & =\frac{\left(-R_{n+2}-1-\sqrt{\alpha_{3}}\right)}{2}, \\
\alpha_{3} & =\left(R_{n+2}+1\right)^{2}-4\left(R_{n+1}+2\right)\left(R_{n}-3\right), \\
\alpha_{4} & =\left(R_{n}-3\right)^{n}+R_{n+5}\left(R_{n+1}+2\right)^{n-1}, \\
\prod_{i=1}^{n}\left(-\varepsilon_{i}^{3}+\varepsilon_{i}+1\right) & =-\frac{1}{2}\left(R_{n+2} R_{n-5}-R_{2 n-3}-2 R_{n+4}-4\right) .
\end{aligned}
$$

Therefore,

$$
\operatorname{det} \mathbf{D}=\frac{2\left[\alpha_{4}+\left(R_{n}-3\right)\left(\alpha_{1}^{n-1}+\alpha_{2}^{n-1}\right)-\left(\alpha_{1}^{n}+\alpha_{2}^{n}\right)\right]}{-R_{n+2} R_{n-5}+R_{2 n-3}+2 R_{n+4}+4} .
$$

The proof is completed.

Corollary 7. Let $\mathbf{E}=R L M F L \operatorname{circfr}\left(R_{1}, R_{2}, \ldots, R_{n}\right)$. If $n \equiv$ $0(\bmod 4)$ or $n \equiv 1(\bmod 4)$, then

$$
\operatorname{det} \mathbf{E}=\frac{2\left[\alpha_{4}+\left(R_{n}-3\right)\left(\alpha_{1}^{n-1}+\alpha_{2}^{n-1}\right)-\left(\alpha_{1}^{n}+\alpha_{2}^{n}\right)\right]}{-R_{n+2} R_{n-5}+R_{2 n-3}+2 R_{n+4}+4},
$$

and if $n \equiv 2(\bmod 4)$ or $n \equiv 3(\bmod 4)$, then

$$
\operatorname{det} \mathbf{E}=\frac{2\left[\alpha_{4}+\left(R_{n}-3\right)\left(\alpha_{1}^{n-1}+\alpha_{2}^{n-1}\right)-\left(\alpha_{1}^{n}+\alpha_{2}^{n}\right)\right]}{R_{n+2} R_{n-5}-R_{2 n-3}-2 R_{n+4}-4},
$$

where $\alpha_{1}, \alpha_{2}, \alpha_{3}, \alpha_{4}$ are defined the same as Theorem 6.

Proof. Since

$$
\operatorname{det} \widehat{\mathbf{I}}=(-1)^{n(n-1) / 2},
$$

the result can be derived from Theorem 6 and relation (4).

\subsection{Determinants of the RFMLR and RLMFL Circulant} Matrix Involving Padovan Sequence

Theorem 8. Let $\mathbf{F}=\operatorname{RFMLR\operatorname {circ}fr}\left(\mathbb{P}_{1}, \mathbb{P}_{2}, \ldots, \mathbb{P}_{n}\right)$. If $n$ is odd, then

$$
\operatorname{det} \mathbf{F}=\frac{\left(-Q_{1}+Q_{2}+Q_{3}\right)}{\mathscr{Y}_{1}},
$$

and if $n$ is even, then

$$
\operatorname{det} \mathbf{F}=\frac{\left(Q_{1}-Q_{2}-Q_{3}\right)}{\mathscr{Y}_{1}},
$$

where

$$
\begin{aligned}
& \mathbb{Q}_{1}=\mathbb{P}_{n}^{n}\left(Q_{n}^{2}-Q_{2 n}-2 Q_{n+1}\right), \\
& \mathcal{Q}_{2}=\left(\mathbb{P}_{n+1}-1\right) \mathbb{P}_{n}^{n-1}\left(Q_{n-1}^{2}-Q_{2(n-1)}+2 Q_{n-1}\right), \\
& \mathbb{Q}_{3}=2 \mathbb{P}_{n}^{n-1}\left(\mathbb{P}_{n+2} Q_{n}+Q_{n}-3\right)+2\left(\mathbb{P}_{n+1}-1\right)^{n}, \\
& Q_{n}=q_{1}^{n}+q_{2}^{n}+q_{3}^{n},
\end{aligned}
$$

where $q_{1}, q_{2}, q_{3}$ are the roots of the equation $\mathbb{P}_{n} x^{3}+(1+$ $\left.\mathbb{P}_{n-1}\right) x^{2}+\left(1-\mathbb{P}_{n-3}\right) x+1-\mathbb{P}_{n+1}=0$ and $\mathscr{Y}_{1}$ is defined as Theorem 5 .

Proof. The matrix $\mathbf{F}$ has the form

$$
\mathbf{F}=\left(\begin{array}{cccc}
\mathbb{P}_{1} & \mathbb{P}_{2} & \ldots & \mathbb{P}_{n} \\
\mathbb{P}_{n} & \mathbb{P}_{1}-\mathbb{P}_{n} & \cdots & \mathbb{P}_{n-1} \\
\vdots & \vdots & \ddots & \vdots \\
\mathbb{P}_{3} & \mathbb{P}_{4}-\mathbb{P}_{3} & \cdots & \mathbb{P}_{2} \\
\mathbb{P}_{2} & \mathbb{P}_{3}-\mathbb{P}_{2} & \ldots & \mathbb{P}_{1}-\mathbb{P}_{n}
\end{array}\right)
$$


The determinant of $\mathbf{F}$ is

$$
\begin{aligned}
\operatorname{det} \mathbf{F} & =\prod_{i=1}^{n}\left(\mathbb{P}_{1}+\mathbb{P}_{2} \varepsilon_{i}+\cdots+\mathbb{P}_{n} \varepsilon_{i}^{n-1}\right) \\
& =\prod_{i=1}^{n} \sum_{k=1}^{n} \sum_{j=1}^{3} a_{j} r_{j}^{k} \varepsilon_{i}^{k-1} \\
& =\prod_{i=1}^{n} \sum_{j=1}^{3} \frac{a_{j} r_{j}\left(1-r_{j}^{n} \varepsilon_{i}^{n}\right)}{1-r_{j} \varepsilon_{i}} \\
& =\prod_{i=1}^{n}\left[\frac{\mathbb{P}_{n} \varepsilon_{i}^{3}+\left(1+\mathbb{P}_{n-1}\right) \varepsilon_{i}^{2}+\left(1-\mathbb{P}_{n-3}\right) \varepsilon_{i}}{-\varepsilon_{i}^{3}-\varepsilon_{i}^{2}+1}\right. \\
& \left.\quad+\frac{1-\mathbb{P}_{n+1}}{-\varepsilon_{i}^{3}-\varepsilon_{i}^{2}+1}\right]
\end{aligned}
$$

from Lemma 3 and the Binet form (12) and (10).

Using Lemma 4 and recurrence (7), we obtain

$$
\begin{aligned}
\prod_{i=1}^{n}\left[\mathbb{P}_{n} \varepsilon_{i}^{3}+(1+\right. & \left.\left.\mathbb{P}_{n-1}\right) \varepsilon_{i}^{2}+\left(1-\mathbb{P}_{n-3}\right) \varepsilon_{i}+1-\mathbb{P}_{n+1}\right] \\
=\left(-\mathbb{P}_{n}\right)^{n-1}[ & {\left[\mathbb{P}_{n} Q_{n}^{2}+\left(1-\mathbb{P}_{n+1}\right) Q_{n-1}^{2}-\mathbb{P}_{n} Q_{2 n}\right.} \\
& -\left(1-\mathbb{P}_{n+1}\right) Q_{2(n-1)}-2 \mathbb{P}_{n} Q_{n+1} \\
& \left.-2\left(1+\mathbb{P}_{n+2}\right) Q_{n}+2\left(1-\mathbb{P}_{n+1}\right) Q_{n-1}\right] \times 2^{-1} \\
+ & 3\left(-\mathbb{P}_{n}\right)^{n-1}+\left(1-\mathbb{P}_{n+1}\right)^{n},
\end{aligned}
$$

where $Q_{n}=q_{1}^{n}+q_{2}^{n}+q_{3}^{n}, q_{1}, q_{2}, q_{3}$ are the roots of the equation $\mathbb{P}_{n} x^{3}+\left(1+\mathbb{P}_{n-1}\right) x^{2}+\left(1-\mathbb{P}_{n-3}\right) x+1-\mathbb{P}_{n+1}=0$. According to (33), we have the following results: if $n$ is odd, then

$$
\operatorname{det} \mathbf{F}=\frac{\left(-Q_{1}+Q_{2}+Q_{3}\right)}{\mathscr{Y}_{1}},
$$

and if $n$ is even, then

$$
\operatorname{det} \mathbf{F}=\frac{\left(Q_{1}-Q_{2}-Q_{3}\right)}{\mathscr{Y}_{1}},
$$

where

$$
\begin{aligned}
& Q_{1}=\mathbb{P}_{n}^{n}\left(Q_{n}^{2}-Q_{2 n}-2 Q_{n+1}\right), \\
& Q_{2}=\left(\mathbb{P}_{n+1}-1\right) \mathbb{P}_{n}^{n-1}\left(Q_{n-1}^{2}-Q_{2(n-1)}+2 Q_{n-1}\right), \\
& Q_{3}=2 \mathbb{P}_{n}^{n-1}\left(\mathbb{P}_{n+2} Q_{n}+Q_{n}-3\right)+2\left(\mathbb{P}_{n+1}-1\right)^{n},
\end{aligned}
$$

and $\mathscr{Y}_{1}$ is defined as Theorem 5 .

Theorem 9. Let $\mathbf{G}=R F M L R \operatorname{circfr}\left(\mathbb{P}_{n}, \ldots, \mathbb{P}_{1}\right)$. If $n$ is odd, then

$$
\operatorname{det} \mathbf{G}=\frac{\mathscr{Z}_{1}+\mathscr{E}_{2}-\mathscr{Z}_{3}+2\left(\mathbb{P}_{n}-1\right)^{n}}{-R_{n+2} R_{n-5}+R_{2 n-3}+2 R_{n+4}+4},
$$

and if $n$ is even, then

$$
\operatorname{det} \mathbf{G}=\frac{\mathscr{Z}_{1}+\mathscr{Z}_{2}-\mathscr{Z}_{3}-2\left(\mathbb{P}_{n}-1\right)^{n}}{R_{n+2} R_{n-5}-R_{2 n-3}-2 R_{n+4}-4},
$$

where

$$
\begin{aligned}
& \mathscr{Z}_{1}=\left(\mathbb{P}_{n}-1\right)\left(Z_{n-1}^{2}-Z_{2(n-1)}+2 Z_{n-1}\right), \\
& \mathscr{Z}_{2}=Z_{n}^{2}-Z_{2 n}-2 Z_{n+1}, \\
& \mathscr{Z}_{3}=2\left[\left(1+\mathbb{P}_{n+1}\right) Z_{n}-\mathbb{P}_{n+5}\right], \\
& Z_{n}=z_{1}^{n}+z_{2}^{n}+z_{3}^{n},
\end{aligned}
$$

where $z_{1}, z_{2}, z_{3}$ are the roots of the equation $x^{3}+\mathbb{P}_{n+1} x^{2}+$ $\mathbb{P}_{n+2} x+\mathbb{P}_{n}-1=0$.

Proof. The matrix $\mathbf{G}$ has the form

$$
\mathbf{G}=\left(\begin{array}{cccc}
\mathbb{P}_{n} & \mathbb{P}_{n-1} & \ldots & \mathbb{P}_{1} \\
\mathbb{P}_{1} & \mathbb{P}_{n}-\mathbb{P}_{1} & \cdots & \mathbb{P}_{2} \\
\vdots & \vdots & \ddots & \vdots \\
\mathbb{P}_{n-2} & \mathbb{P}_{n-3}-\mathbb{P}_{n-2} & \cdots & \mathbb{P}_{n-1} \\
\mathbb{P}_{n-1} & \mathbb{P}_{n-2}-\mathbb{P}_{n-1} & \cdots & \mathbb{P}_{n}-\mathbb{P}_{n-1}
\end{array}\right)
$$

According to Lemma 3 and the Binet form (12) and (10), we have

$$
\begin{aligned}
\operatorname{det} \mathbf{G} & =\prod_{i=1}^{n}\left(\mathbb{P}_{n}+\mathbb{P}_{n-1} \varepsilon_{i}+\cdots+\mathbb{P}_{1} \varepsilon_{i}^{n-1}\right) \\
& =\prod_{i=1}^{n} \sum_{k=0}^{n-1} \sum_{j=1}^{3} a_{j} r_{j}^{n-k} \varepsilon_{i}^{k} \\
& =\prod_{i=1}^{n} \sum_{j=1}^{3} \frac{a_{j} r_{j}^{n+1}-a_{j} r_{j} \varepsilon_{i}^{n}}{r_{j}-\varepsilon_{i}} \\
& =\prod_{i=1}^{n} \frac{\varepsilon_{i}^{3}+\mathbb{P}_{n+1} \varepsilon_{i}^{2}+\mathbb{P}_{n+2} \varepsilon_{i}+\mathbb{P}_{n}-1}{-\varepsilon_{i}^{3}+\varepsilon_{i}+1} .
\end{aligned}
$$

Using Lemma 4 and (12), we obtain

$$
\begin{gathered}
\prod_{i=1}^{n}\left(\varepsilon_{i}^{3}+\mathbb{P}_{n+1} \varepsilon_{i}^{2}+\mathbb{P}_{n+2} \varepsilon_{i}+\mathbb{P}_{n}-1\right) \\
=(-1)^{n-1}\left[\left(\mathbb{P}_{n}-1\right)\left(Z_{n-1}^{2}-Z_{2(n-1)}+2 Z_{n-1}\right)\right. \\
+Z_{n}^{2}-Z_{2 n}-2 Z_{n+1}-2\left(1+\mathbb{P}_{n+1}\right) Z_{n} \\
\left.+2 \mathbb{P}_{n+5}\right] \times 2^{-1}+\left(\mathbb{P}_{n}-1\right)^{n},
\end{gathered}
$$

where $Z_{n}=z_{1}^{n}+z_{2}^{n}+z_{3}^{n}, z_{1}, z_{2}, z_{3}$ are the roots of the equation $x^{3}+\mathbb{P}_{n+1} x^{2}+\mathbb{P}_{n+2} x+\mathbb{P}_{n}-1=0$. Employing (43), we have the following results: if $n$ is odd, then

$$
\operatorname{det} \mathbf{G}=\frac{\mathscr{Z}_{1}+\mathscr{Z}_{2}-\mathscr{Z}_{3}+2\left(\mathbb{P}_{n}-1\right)^{n}}{-R_{n+2} R_{n-5}+R_{2 n-3}+2 R_{n+4}+4},
$$


and if $n$ is even, then

$$
\operatorname{det} \mathbf{G}=\frac{\mathscr{Z}_{1}+\mathscr{Z}_{2}-\mathscr{Z}_{3}-2\left(\mathbb{P}_{n}-1\right)^{n}}{R_{n+2} R_{n-5}-R_{2 n-3}-2 R_{n+4}-4},
$$

where

$$
\begin{aligned}
& \mathscr{Z}_{1}=\left(\mathbb{P}_{n}-1\right)\left(Z_{n-1}^{2}-Z_{2(n-1)}+2 Z_{n-1}\right), \\
& \mathscr{Z}_{2}=Z_{n}^{2}-Z_{2 n}-2 Z_{n+1}, \\
& \mathscr{Z}_{3}=2\left[\left(1+\mathbb{P}_{n+1}\right) Z_{n}-\mathbb{P}_{n+5}\right] .
\end{aligned}
$$

Corollary 10. Let $\mathbf{H}=R L M F L \operatorname{circfr}\left(\mathbb{P}_{1}, \ldots, \mathbb{P}_{n}\right)$. If $n \equiv$ $0(\bmod 4)$, then

$$
\operatorname{det} \mathbf{H}=\frac{\mathscr{E}_{1}+\mathscr{E}_{2}-\mathscr{Z}_{3}-2\left(\mathbb{P}_{n}-1\right)^{n}}{R_{n+2} R_{n-5}-R_{2 n-3}-2 R_{n+4}-4},
$$

and if $n \equiv 1(\bmod 4)$, then

$$
\operatorname{det} \mathbf{H}=\frac{\mathscr{Z}_{1}+\mathscr{Z}_{2}-\mathscr{E}_{3}+2\left(\mathbb{P}_{n}-1\right)^{n}}{-R_{n+2} R_{n-5}+R_{2 n-3}+2 R_{n+4}+4},
$$

and if $n \equiv 2(\bmod 4)$, then

$$
\operatorname{det} \mathbf{H}=\frac{-\mathscr{E}_{1}-\mathscr{Z}_{2}+\mathscr{E}_{3}+2\left(\mathbb{P}_{n}-1\right)^{n}}{R_{n+2} R_{n-5}-R_{2 n-3}-2 R_{n+4}-4},
$$

and if $n \equiv 3(\bmod 4)$, then

$$
\operatorname{det} \mathbf{H}=\frac{-\mathscr{E}_{1}-\mathscr{E}_{2}+\mathscr{E}_{3}-2\left(\mathbb{P}_{n}-1\right)^{n}}{-R_{n+2} R_{n-5}+R_{2 n-3}+2 R_{n+4}+4},
$$

where $\mathscr{Z}_{1}, \mathscr{Z}_{2}, \mathscr{Z}_{3}$ are defined the same as Theorem 9.

Proof. The theorem can be proved by using Theorem 9 and relation (4).

\subsection{Determinants of the RFMLR and RLMFL Circulant} Matrix Involving Tribonacci Numbers

Theorem 11. Let $\mathbf{J}=R F M L R \operatorname{circfr}\left(T_{1}, T_{2}, \ldots, T_{n}\right)$. If $n$ is odd then

$$
\operatorname{det} \mathbf{J}=\frac{\left(-\mathscr{U}_{1}-\mathscr{U}_{2}-\mathscr{U}_{3}-2\left(1-T_{n+1}\right)^{n}\right)}{\mathscr{V}_{1}}
$$

and if $n$ is even, then

$$
\operatorname{det} \mathbf{J}=\frac{\left(\mathscr{U}_{1}+\mathscr{U}_{2}+\mathscr{U}_{3}-2\left(1-T_{n+1}\right)^{n}\right)}{\mathscr{V}_{1}},
$$

where

$$
\begin{aligned}
\mathcal{U}_{1}= & T_{n}^{n}\left(U_{n}^{2}-U_{2 n}-2 U_{n+1}-2 U_{n}\right), \\
\mathcal{U}_{2}= & 2 T_{n}^{n-1}\left(1-T_{n-1} U_{n}\right), \\
\mathcal{U}_{3}= & T_{n}^{n-1}\left(1-T_{n+1}\right)\left(U_{n-1}^{2}-U_{2(n-1)}+2 U_{n-1}\right), \\
\mathscr{V}_{1}= & V_{n}^{2}-V_{n-1}^{2}-V_{2 n}+V_{2(n-1)}-2 V_{n+1} \\
& -4 V_{n}-2 V_{n-1}+2, \\
U_{n}= & u_{1}^{n}+u_{2}^{n}+u_{3}^{n}, \\
V_{n}= & v_{1}^{n}+v_{2}^{n}+v_{3}^{n},
\end{aligned}
$$

where $u_{1}, u_{2}, u_{3}$ and $v_{1}, v_{2}, v_{3}$ are the roots of the equations $T_{n} x^{3}+T_{n-1} x^{2}+T_{n-2} x+1-T_{n+1}=0, x^{3}+x^{2}+x-1=0$, respectively.

Proof. Obviously, $\mathrm{J}$ has the form

$$
\mathbf{J}=\left(\begin{array}{cccc}
T_{1} & T_{2} & \ldots & T_{n} \\
T_{n} & T_{1}-T_{n} & \ldots & T_{n-1} \\
\vdots & \vdots & \ddots & \vdots \\
T_{3} & T_{4}-T_{3} & \ldots & T_{2} \\
T_{2} & T_{3}-T_{2} & \ldots & T_{1}-T_{n}
\end{array}\right)
$$

According to Lemma 3 and the Binet form (16) and (14), we have

$$
\begin{aligned}
\operatorname{det} \mathbf{J} & =\prod_{i=1}^{n}\left(T_{1}+T_{2} \varepsilon_{i}+\cdots+T_{n} \varepsilon_{i}^{n-1}\right) \\
& =\prod_{i=1}^{n} \sum_{k=1}^{n} \sum_{j=1}^{3} b_{j} t_{j}^{k} \varepsilon_{i}^{k-1} \\
& =\prod_{i=1}^{n} \sum_{i=1}^{3} \frac{b_{j} t_{j}\left(1-t_{j}^{n} \varepsilon_{i}^{n}\right)}{1-t_{j} \varepsilon_{i}} \\
& =\prod_{i=1}^{n} \frac{T_{n} \varepsilon_{i}^{3}+T_{n-1} \varepsilon_{i}^{2}+T_{n-2} \varepsilon_{i}+1-T_{n+1}}{-\varepsilon_{i}^{3}-\varepsilon_{i}^{2}-\varepsilon_{i}+1} .
\end{aligned}
$$

From Lemma 4 it follows that

$$
\begin{aligned}
\prod_{i=1}^{n}( & \left.T_{n} \varepsilon_{i}^{3}+T_{n-1} \varepsilon_{i}^{2}+T_{n-2} \varepsilon_{i}+1-T_{n+1}\right) \\
= & \frac{1}{2}\left(-T_{n}\right)^{n}\left(-U_{n}^{2}+U_{2 n}+2 U_{n+1}+2 U_{n}\right) \\
& +\left(-T_{n}\right)^{n-1}\left(1-T_{n-1} U_{n}\right)+\frac{1}{2}\left(-T_{n}\right)^{n-1}\left(1-T_{n+1}\right) \\
& \times\left(U_{n-1}^{2}-U_{2(n-1)}+2 U_{n-1}\right)+\left(1-T_{n+1}\right)^{n},
\end{aligned}
$$


where $U_{n}=u_{1}^{n}+u_{2}^{n}+u_{3}^{n}, u_{1}, u_{2}, u_{3}$ are the roots of the equation $T_{n} x^{3}+T_{n-1} x^{2}+T_{n-2} x+1-T_{n+1}=0$. And

$$
\begin{aligned}
\prod_{i=1}^{n}\left(-\varepsilon_{i}^{3}-\varepsilon_{i}^{2}-\varepsilon_{i}+1\right)=\frac{1}{2}( & -V_{n}^{2}+V_{n-1}^{2}+V_{2 n} \\
& -V_{2(n-1)}+2 V_{n+1}, \\
& \left.+4 V_{n}+2 V_{n-1}-2\right),
\end{aligned}
$$

where $V_{n}=v_{1}^{n}+v_{2}^{n}+v_{3}^{n}, v_{1}, v_{2}, v_{3}$ are the roots of the equation $x^{3}+x^{2}+x-1=0$. Consequently, we have the following results: if $n$ is odd, then

$$
\operatorname{det} \mathbf{J}=\frac{\left(-\mathscr{U}_{1}-\mathscr{U}_{2}-\mathscr{U}_{3}-2\left(1-T_{n+1}\right)^{n}\right)}{\mathscr{V}_{1}},
$$

and if $n$ is even, then

$$
\operatorname{det} \mathbf{J}=\frac{\left(\mathscr{U}_{1}+\mathscr{U}_{2}+\mathscr{U}_{3}-2\left(1-T_{n+1}\right)^{n}\right)}{\mathscr{V}_{1}},
$$

where

$$
\begin{aligned}
\mathscr{U}_{1}= & T_{n}^{n}\left(U_{n}^{2}-U_{2 n}-2 U_{n+1}-2 U_{n}\right), \\
\mathcal{U}_{2}= & 2 T_{n}^{n-1}\left(1-T_{n-1} U_{n}\right), \\
\mathscr{U}_{3}= & T_{n}^{n-1}\left(1-T_{n+1}\right)\left(U_{n-1}^{2}-U_{2(n-1)}+2 U_{n-1}\right), \\
\mathscr{V}_{1}= & V_{n}^{2}-V_{n-1}^{2}-V_{2 n}+V_{2(n-1)}-2 V_{n+1} \\
& -4 V_{n}-2 V_{n-1}+2 .
\end{aligned}
$$

The proof is completed.

Theorem 12. Let $\mathbf{K}=R F M L R \operatorname{circfr}\left(T_{n}, \ldots, T_{1}\right)$. If $n$ is odd, then

$$
\operatorname{det} \mathbf{K}=\frac{\left(-\mathscr{W}_{1}-\mathscr{W}_{2}+\mathscr{W}_{3}\right)}{\mathscr{L}_{1}},
$$

and if $n$ is even, then

$$
\operatorname{det} \mathbf{K}=\frac{\left(\mathscr{W}_{1}+\mathscr{W}_{2}-\mathscr{W}_{3}\right)}{\mathscr{L}_{1}},
$$

where

$$
\begin{aligned}
\mathscr{W}_{1}= & W_{n}^{2}-W_{2 n}-2 W_{n+1}, \\
\mathscr{W}_{2}= & T_{n}\left(W_{n-1}^{2}-W_{2(n-1)}+2 W_{n-1}\right), \\
\mathscr{W}_{3}= & 2\left(T_{n+1} W_{n}-T_{n+2}-T_{n}+T_{n}^{n}\right), \\
\mathscr{L}_{1}= & \mathbb{L}_{n}^{2}-\mathbb{L}_{n-1}^{2}-\mathbb{L}_{2 n}+\mathbb{L}_{2(n-1)}-2 \mathbb{L}_{n+1} \\
& -2 \mathbb{L}_{n-1}-6, \\
W_{n}= & w_{1}^{n}+w_{2}^{n}+w_{3}^{n},
\end{aligned}
$$

where $w_{1}, w_{2}, w_{3}$ are the roots of the equation $x^{3}+\left(T_{n+1}-\right.$ 1) $x^{2}+\left(T_{n+2}-T_{n+1}\right) x+T_{n}=0$.
Proof. The matrix $\mathbf{K}$ has the form

$$
\mathbf{K}=\left(\begin{array}{cccc}
T_{n} & T_{n-1} & \ldots & T_{1} \\
T_{1} & T_{n}-T_{1} & \ldots & T_{2} \\
\vdots & \vdots & \ddots & \vdots \\
T_{n-2} & T_{n-3}-T_{n-2} & \ldots & T_{n-1} \\
T_{n-1} & T_{n-2}-T_{n-1} & \ldots & T_{n}-T_{1}
\end{array}\right)
$$

According to Lemma 3 and the Binet form (16) and (14), we have

$$
\begin{aligned}
\operatorname{det} \mathbf{K} & =\prod_{i=1}^{n}\left(T_{n}+T_{n-1} \varepsilon_{i}+\cdots+T_{1} \varepsilon_{i}^{n-1}\right) \\
& =\prod_{i=1}^{n} \sum_{k=0}^{n-1} \sum_{j=1}^{3} b_{j} t_{j}^{n-k} \varepsilon_{i}^{k} \\
& =\prod_{i=1}^{n} \sum_{j=1}^{3} \frac{b_{j} t_{j}^{n+1}-b_{j} t_{j} \varepsilon_{i}^{n}}{t_{j}-\varepsilon_{i}} \\
& =\prod_{i=1}^{n} \frac{\varepsilon_{i}^{3}+\left(T_{n+1}-1\right) \varepsilon_{i}^{2}+\left(T_{n+2}-T_{n+1}\right) \varepsilon_{i}+T_{n}}{-\varepsilon_{i}^{3}+\varepsilon_{i}^{2}+\varepsilon_{i}+1} .
\end{aligned}
$$

Considering Lemma 4 and (17), we obtain

$$
\begin{gathered}
\prod_{i=1}^{n}\left[\varepsilon_{i}^{3}+\left(T_{n+1}-1\right) \varepsilon_{i}^{2}+\left(T_{n+2}-T_{n+1}\right) \varepsilon_{i}+T_{n}\right] \\
=\frac{1}{2}(-1)^{n-1}\left[W_{n}^{2}-W_{2 n}-2 W_{n+1}-2 W_{n}\right. \\
\left.\quad+T_{n}\left(W_{n-1}^{2}-W_{2(n-1)}+2 W_{n-1}\right)\right] \\
\quad+(-1)^{n-1}\left(-T_{n+1} W_{n}+W_{n}+T_{n+2}+T_{n}\right)+T_{n}^{n},
\end{gathered}
$$

where $W_{n}=w_{1}^{n}+w_{2}^{n}+w_{3}^{n}, w_{1}, w_{2}, w_{3}$ are the roots of the equation $x^{3}+\left(T_{n+1}-1\right) x^{2}+\left(T_{n+2}-T_{n+1}\right) x+T_{n}=0$. And

$$
\begin{aligned}
\prod_{i=1}^{n}\left(-\varepsilon_{i}^{3}+\varepsilon_{i}^{2}+\varepsilon_{i}+1\right)= & \left(-\mathbb{L}_{n}^{2}+\mathbb{L}_{n-1}^{2}+\mathbb{L}_{2 n}\right. \\
& \left.-\mathbb{L}_{2(n-1)}+2 \mathbb{L}_{n+1}+2 \mathbb{L}_{n-1}+6\right) \\
& \times 2^{-1} .
\end{aligned}
$$

Consequently, if $n$ is odd, then

$$
\operatorname{det} \mathbf{K}=\frac{\left(-\mathscr{W}_{1}-\mathscr{W}_{2}+\mathscr{W}_{3}\right)}{\mathscr{L}_{1}},
$$

and if $n$ is even, then

$$
\operatorname{det} \mathbf{K}=\frac{\left(\mathscr{W}_{1}+\mathscr{W}_{2}-\mathscr{W}_{3}\right)}{\mathscr{L}_{1}},
$$


where

$$
\begin{aligned}
\mathscr{W}_{1} & =W_{n}^{2}-W_{2 n}-2 W_{n+1}, \\
\mathscr{W}_{2} & =T_{n}\left(W_{n-1}^{2}-W_{2(n-1)}+2 W_{n-1}\right), \\
\mathscr{W}_{3} & =2\left(T_{n+1} W_{n}-T_{n+2}-T_{n}+T_{n}^{n}\right), \\
\mathscr{L}_{1} & =\mathbb{L}_{n}^{2}-\mathbb{L}_{n-1}^{2}-\mathbb{L}_{2 n}+\mathbb{L}_{2(n-1)} \\
& -2 \mathbb{L}_{n+1}-2 \mathbb{L}_{n-1}-6 .
\end{aligned}
$$

Corollary 13. Let $\mathbf{L}=R L M F L \operatorname{circfr}\left(T_{1}, T_{2}, \ldots, T_{n}\right)$. If $n \equiv$ $0(\bmod 4)$, then

$$
\operatorname{det} \mathbf{L}=\frac{\left(\mathscr{W}_{1}+\mathscr{W}_{2}-\mathscr{W}_{3}\right)}{\mathscr{L}_{1}},
$$

and if $n \equiv 1(\bmod 4)$, then

$$
\operatorname{det} \mathbf{L}=\frac{\left(-\mathscr{W}_{1}-\mathscr{W}_{2}+\mathscr{W}_{3}\right)}{\mathscr{L}_{1}},
$$

and if $n \equiv 2(\bmod 4)$, then

$$
\operatorname{det} \mathbf{L}=\frac{\left(-\mathscr{W}_{1}-\mathscr{W}_{2}+\mathscr{W}_{3}\right)}{\mathscr{L}_{1}},
$$

and if $n \equiv 3(\bmod 4)$, then

$$
\operatorname{det} \mathbf{L}=\frac{\left(\mathscr{W}_{1}+\mathscr{W}_{2}-\mathscr{W}_{3}\right)}{\mathscr{L}_{1}},
$$

where $\mathscr{W}_{1}, \mathscr{W}_{2}, \mathscr{W}_{3}, \mathscr{L}_{1}$ are defined as Theorem 12.

Proof. The theorem can be proved by using Theorem 12 and relation (4).

\subsection{Determinants of the RFMLR and RLMFL Circulant} Matrix Involving Generalized Lucas Numbers

Theorem 14. Let $\mathbf{M}=R F M L R \operatorname{circfr}\left(\mathbb{L}_{1}, \ldots, \mathbb{L}_{n}\right)$. If $n$ is odd, then

$$
\operatorname{det} \mathbf{M}=\frac{\left(-\mathcal{S}_{1}+\mathcal{S}_{2}-\mathcal{S}_{3}-2\left(1-\mathbb{\mathbb { L }}_{n+1}\right)^{n}\right)}{\mathscr{V}_{1}},
$$

and if $n$ is even, then

$$
\operatorname{det} \mathbf{M}=\frac{\left(\mathcal{S}_{1}-\mathcal{S}_{2}+\mathcal{S}_{3}-2\left(1-\mathbb{\mathbb { L }}_{n+1}\right)^{n}\right)}{\mathscr{V}_{1}},
$$

where

$$
\begin{aligned}
& \mathcal{S}_{1}=\mathbb{L}_{n}^{n}\left(S_{n}^{2}-S_{2 n}-2 S_{n+1}-2 S_{n}\right), \\
& \mathcal{S}_{2}=2 \mathbb{L}_{n}^{n-1}\left(\mathbb{L}_{n-1} S_{n}+3 S_{n}-6\right), \\
& \mathcal{S}_{3}=\left(1-\mathbb{L}_{n+1}\right)\left(S_{n-1}^{2}-S_{2(n-1)}+2 S_{n-1}\right), \\
& S_{n}=s_{1}^{n}+s_{2}^{n}+s_{3}^{n},
\end{aligned}
$$

where $s_{1}, s_{2}, s_{3}$ are the roots of the equation $\mathbb{L}_{n} x^{3}+\left(\mathbb{L}_{n-1}+3\right) x^{2}+$ $\left(\mathbb{L}_{n-2}+2\right) x+1-\mathbb{L}_{n+1}=0$, and $\mathscr{V}_{1}$ is defined as Theorem 11 .
Proof. The matrix $\mathbf{M}$ has the form

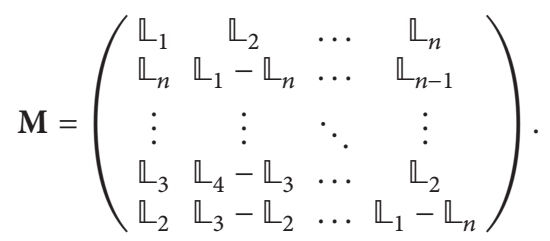

According to Lemma 3 and the Binet form (17) and (14), we have

$$
\begin{aligned}
\operatorname{det} \mathbf{M}=\prod_{i=1}^{n}\left(\mathbb{L}_{1}+\mathbb{L}_{2} \varepsilon_{i}+\cdots+\mathbb{L}_{n} \varepsilon_{i}^{n-1}\right) \\
=\prod_{i=1}^{n} \sum_{k=1}^{n} \sum_{j=1}^{3} t_{j}^{k} \varepsilon_{i}^{k-1} \\
=\prod_{i=1}^{n} \sum_{j=1}^{3} \frac{t_{j}\left(1-t_{j}^{n} \varepsilon_{i}^{n}\right)}{1-t_{j}^{n} \varepsilon_{i}} \\
=\prod_{i=1}^{n}\left[\frac{\mathbb{L}_{n} \varepsilon_{i}^{3}+\left(\mathbb{L}_{n-1}+3\right) \varepsilon_{i}^{2}+\left(\mathbb{L}_{n-2}+2\right) \varepsilon_{i}}{-\varepsilon_{i}^{3}-\varepsilon_{i}^{2}-\varepsilon_{i}+1}\right. \\
\left.\quad+\frac{1-\mathbb{L}_{n+1}}{-\varepsilon_{i}^{3}-\varepsilon_{i}^{2}-\varepsilon_{i}+1}\right] .
\end{aligned}
$$

From Lemma 4 and (17), we obtain

$$
\begin{aligned}
& \prod_{i=1}^{n}\left[\mathbb{L}_{n} \varepsilon_{i}^{3}+\left(\mathbb{L}_{n-1}+3\right) \varepsilon_{i}^{2}+\left(\mathbb{L}_{n-2}+2\right) \varepsilon_{i}+1-\mathbb{L}_{n+1}\right] \\
& =\frac{1}{2}\left(-\mathbb{L}_{n}\right)^{n}\left(-S_{n}^{2}+S_{2 n}+2 S_{n+1}+2 S_{n}\right)+\left(-\mathbb{L}_{n}\right)^{n-1} \\
& \times\left(-\mathbb{L}_{n-1} S_{n}-3 S_{n}+6\right)+\frac{1}{2}\left(-\mathbb{L}_{n}\right)^{n-1}\left(1-\mathbb{L}_{n+1}\right) \\
& \times\left(S_{n-1}^{2}-S_{2(n-1)}+2 S_{n-1}\right)+\left(1-\mathbb{L}_{n+1}\right)^{n},
\end{aligned}
$$

where $S_{n}=s_{1}^{n}+s_{2}^{n}+s_{3}^{n}, s_{1}, s_{2}, s_{3}$ are the roots of the equation $\mathbb{L}_{n} x^{3}+\left(\mathbb{L}_{n-1}+3\right) x^{2}+\left(\mathbb{L}_{n-2}+2\right) x+1-\mathbb{L}_{n+1}=0$. And

$$
\begin{aligned}
\prod_{i=1}^{n}\left(-\varepsilon_{i}^{3}-\varepsilon_{i}^{2}-\varepsilon_{i}+1\right)=\frac{1}{2}( & -V_{n}^{2}+V_{n-1}^{2}+V_{2 n} \\
- & V_{2(n-1)}+2 V_{n+1} \\
& \left.+4 V_{n}+2 V_{n-1}-2\right),
\end{aligned}
$$

where $V_{n}=v_{1}^{n}+v_{2}^{n}+v_{3}^{n}, v_{1}, v_{2}, v_{3}$ are the roots of the equation $x^{3}+x^{2}+x-1=0$. Hence, if $n$ is odd, then

$$
\operatorname{det} \mathbf{M}=\frac{-\mathcal{S}_{1}+\mathcal{S}_{2}-\mathcal{S}_{3}-2\left(1-\mathbb{L}_{n+1}\right)^{n}}{\mathscr{V}_{1}},
$$

and if $n$ is even, then

$$
\operatorname{det} \mathbf{M}=\frac{\mathcal{S}_{1}-\mathcal{S}_{2}+\mathcal{S}_{3}-2\left(1-\mathbb{L}_{n+1}\right)^{n}}{\mathscr{V}_{1}},
$$


where

$$
\begin{aligned}
& \mathcal{S}_{1}=\mathbb{L}_{n}^{n}\left(S_{n}^{2}-S_{2 n}-2 S_{n+1}-2 S_{n}\right), \\
& \mathcal{S}_{2}=2 \mathbb{L}_{n}^{n-1}\left(\mathbb{L}_{n-1} S_{n}+3 S_{n}-6\right), \\
& \mathcal{S}_{3}=\left(1-\mathbb{L}_{n+1}\right)\left(S_{n-1}^{2}-S_{2(n-1)}+2 S_{n-1}\right),
\end{aligned}
$$

and $\mathscr{V}_{1}$ is defined as Theorem 11.

Theorem 15. Let $\mathbf{N}=R F M L R \operatorname{circfr}\left(\mathbb{L}_{n}, \ldots, \mathbb{L}_{1}\right)$. If $n$ is odd, then

$$
\operatorname{det} \mathbf{N}=\frac{\left(-\mathscr{T}_{1}+\mathscr{T}_{2}-\mathscr{T}_{3}-2\left(\mathbb{L}_{n}-3\right)^{n}\right)}{\mathscr{L}_{1}},
$$

and if $n$ is even, then

$$
\operatorname{det} \mathbf{N}=\frac{\left(\mathscr{T}_{1}-\mathscr{T}_{2}+\mathscr{T}_{3}-2\left(\mathbb{L}_{n}-3\right)^{n}\right)}{\mathscr{L}_{1}},
$$

where

$$
\begin{aligned}
\mathscr{T}_{1} & =\Gamma_{n}^{2}-\Gamma_{2 n}-2 \Gamma_{n+1}, \\
\mathscr{T}_{2} & =2\left(\mathbb{L}_{n+1}+2\right) \Gamma_{n}-2\left(\mathbb{L}_{n+2}+\mathbb{L}_{n}\right), \\
\mathscr{T}_{3} & =\left(\mathbb{L}_{n}-3\right)\left(\Gamma_{n-1}^{2}-\Gamma_{2(n-1)}+2 \Gamma_{n-1}\right), \\
\Gamma_{n} & =\gamma_{1}^{n}+\gamma_{2}^{n}+\gamma_{3}^{n},
\end{aligned}
$$

where $\gamma_{1}, \gamma_{2}, \gamma_{3}$ are the roots of the equation $x^{3}+\left(\mathbb{L}_{n+1}+1\right) x^{2}+$ $\left(\mathbb{L}_{n+2}-\mathbb{L}_{n+1}+1\right) x+\mathbb{L}_{n}-3=0$, and $\mathscr{L}_{1}$ is defined as Theorem 12 .

Proof. The matrix $\mathbf{N}$ has the form

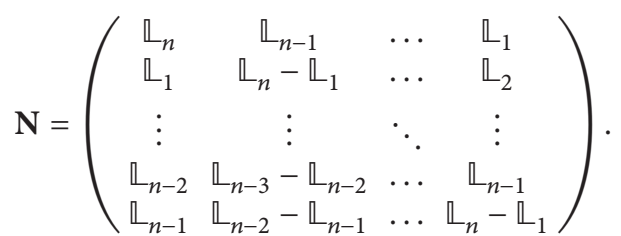

According to Lemma 3, (17), and (14), we have

$$
\begin{aligned}
\operatorname{det} \mathbf{N}= & \prod_{i=1}^{n}\left(\mathbb{L}_{n}+\mathbb{L}_{n-1} \varepsilon_{i}+\cdots+\mathbb{L}_{1} \varepsilon_{i}^{n-1}\right) \\
& =\prod_{i=1}^{n} \sum_{k=0}^{n-1} \sum_{j=1}^{3} t_{j}^{n-k} \varepsilon_{i}^{k} \\
& =\prod_{i=1}^{n} \sum_{j=1}^{3} \frac{t_{j}^{n+1}-t_{j} \varepsilon_{i}^{n}}{t_{j}-\varepsilon_{i}} \\
& =\prod_{i=1}^{n}\left[\frac{\varepsilon_{i}^{3}+\left(\mathbb{L}_{n+1}+1\right) \varepsilon_{i}^{2}+\mathbb{L}_{n}-3}{-\varepsilon_{i}^{3}+\varepsilon_{i}^{2}+\varepsilon_{i}+1}\right. \\
& \left.+\frac{\left(\mathbb{L}_{n+2}-\mathbb{L}_{n+1}+1\right) \varepsilon_{i}}{-\varepsilon_{i}^{3}+\varepsilon_{i}^{2}+\varepsilon_{i}+1}\right] .
\end{aligned}
$$

By Lemma 4 and the Binet form (17), we obtain

$$
\begin{aligned}
& \prod_{i=1}^{n}\left[\varepsilon_{i}^{3}+\left(\mathbb{L}_{n+1}+1\right) \varepsilon_{i}^{2}+\left(\mathbb{L}_{n+2}-\mathbb{L}_{n+1}+1\right) \varepsilon_{i}\right. \\
& \left.\quad+\mathbb{L}_{n}-3\right] \\
& =\frac{1}{2}(-1)^{n}\left(-\Gamma_{n}^{2}+\Gamma_{2 n}+2 \Gamma_{n+1}+2 \Gamma_{n}\right)+(-1)^{n-1} \\
& \quad \times\left(-\mathbb{L}_{n+1} \Gamma_{n}-\Gamma_{n}+\mathbb{L}_{n+2}+\mathbb{L}_{n}\right)+\frac{1}{2}(-1)^{n-1} \\
& \quad \times\left(\mathbb{L}_{n}-3\right)\left(\Gamma_{n-1}^{2}-\Gamma_{2(n-1)}+2 \Gamma_{n-1}\right)+\left(\mathbb{L}_{n}-3\right)^{n},
\end{aligned}
$$

where $\Gamma_{n}=\gamma_{1}^{n}+\gamma_{2}^{n}+\gamma_{3}^{n}, \gamma_{1}, \gamma_{2}, \gamma_{3}$ are the roots of the equation $x^{3}+\left(\mathbb{L}_{n+1}+1\right) x^{2}+\left(\mathbb{L}_{n+2}-\mathbb{L}_{n+1}+1\right) x+\mathbb{L}_{n}-3=0$. And

$$
\begin{aligned}
\prod_{i=1}^{n}\left(-\varepsilon_{i}^{3}+\varepsilon_{i}^{2}+\varepsilon_{i}+1\right)= & \left(-\mathbb{L}_{n}^{2}+\mathbb{L}_{n-1}^{2}+\mathbb{L}_{2 n}\right. \\
& \left.-\mathbb{L}_{2(n-1)}+2 \mathbb{L}_{n+1}+2 \mathbb{L}_{n-1}+6\right) \\
& \times 2^{-1} .
\end{aligned}
$$

Thus, if $n$ is odd, then

$$
\operatorname{det} \mathbf{N}=\frac{\left(-\mathscr{T}_{1}+\mathscr{T}_{2}-\mathscr{T}_{3}-2\left(\mathbb{L}_{n}-3\right)^{n}\right)}{\mathscr{L}_{1}},
$$

and if $n$ is even, then

$$
\operatorname{det} \mathbf{N}=\frac{\left(\mathscr{T}_{1}-\mathscr{T}_{2}+\mathscr{T}_{3}-2\left(\mathbb{L}_{n}-3\right)^{n}\right)}{\mathscr{L}_{1}},
$$

where

$$
\begin{aligned}
& \mathscr{T}_{1}=\Gamma_{n}^{2}-\Gamma_{2 n}-2 \Gamma_{n+1}, \\
& \mathscr{T}_{2}=2\left(\mathbb{L}_{n+1}+2\right) \Gamma_{n}-2\left(\mathbb{L}_{n+2}+\mathbb{L}_{n}\right), \\
& \mathscr{T}_{3}=\left(\mathbb{L}_{n}-3\right)\left(\Gamma_{n-1}^{2}-\Gamma_{2(n-1)}+2 \Gamma_{n-1}\right),
\end{aligned}
$$

and $\mathscr{L}_{1}$ is defined as Theorem 12

Corollary 16. Let $\mathbf{P}=R L M F L \operatorname{circfr}\left(\mathbb{L}_{1}, \ldots, \mathbb{L}_{n}\right)$. If $n \equiv 0(\bmod$ 4), then

$$
\operatorname{det} \mathbf{P}=\frac{\left(\mathscr{T}_{1}-\mathscr{T}_{2}+\mathscr{T}_{3}-2\left(\mathbb{L}_{n}-3\right)^{n}\right)}{\mathscr{L}_{1}},
$$

and if $n \equiv 1(\bmod 4)$, then

$$
\operatorname{det} \mathbf{P}=\frac{\left(-\mathscr{T}_{1}+\mathscr{T}_{2}-\mathscr{T}_{3}-2\left(\mathbb{L}_{n}-3\right)^{n}\right)}{\mathscr{L}_{1}},
$$

and if $n \equiv 2(\bmod 4)$, then

$$
\operatorname{det} \mathbf{P}=\frac{\left(-\mathscr{T}_{1}+\mathscr{T}_{2}-\mathscr{T}_{3}+2\left(\mathbb{L}_{n}-3\right)^{n}\right)}{\mathscr{L}_{1}},
$$


and if $n \equiv 3(\bmod 4)$, then

$$
\operatorname{det} \mathbf{P}=\frac{\left(\mathscr{T}_{1}-\mathscr{T}_{2}+\mathscr{T}_{3}+2\left(\mathbb{L}_{n}-3\right)^{n}\right)}{\mathscr{L}_{1}},
$$

where $\mathscr{T}_{1}, \mathscr{T}_{2}, \mathscr{T}_{3}, \mathscr{L}_{1}$ are defined as Theorem 15 .

Proof. The theorem can be proved by using Theorem 15 and relation (4).

\section{Conclusions}

The row first-minus-last right (RFMLR) circulant matrices and row last-minus-first left (RLMFL) circulant matrices are two kinds of matrices with specific structure. We explored the determinant problem of these two kinds of matrices when their entries are Perrin, Padovan, Tribonacci, and the generalized Lucas sequences, respectively. On the basis of the inverse factorization of polynomial and the thirdorder recurrence, Binet form, and other properties of these sequences, we present the exact formulae of determinants by some terms of these famous sequences. Chillag has studied some properties and applications of $f(x)$-circulant matrices in [7]. The RFMLR circulant and RLMFL circulant matrices have more explicit structures and better properties than the general $f(x)$-circulant matrices, so they will play more important roles than the general $f(x)$-circulant matrices in some fields of signal encoding, image processing, and so on. That is the reason why we focus our attentions on RFMLR circulant and RLMFL circulant matrices.

\section{Conflict of Interests}

The authors declare that there is no conflict of interests regarding the publication of this paper.

\section{Acknowledgments}

The research was supported by the Development Project of Science \& Technology of Shandong Province (Grant no. 2012GGX10115), the NSFC (Grant no. 11301251), and the AMEP of Linyi University, China.

\section{References}

[1] F. Zanderigo, A. Bertoldo, G. Pillonetto, and C. Cobelli, "Nonlinear stochastic regularization to characterize tissue residue function in bolus-tracking MRI: assessment and comparison with SVD, block-circulant SVD, and Tikhonov," IEEE Transactions on Biomedical Engineering, vol. 56, no. 5, pp. 1287-1297, 2009.

[2] H.-J. Wittsack, A. M. Wohlschläger, E. K. Ritzl et al., "CTperfusion imaging of the human brain: advanced deconvolution analysis using circulant singular value decomposition," Computerized Medical Imaging and Graphics, vol. 32, no. 1, pp. 67-77, 2008.

[3] W. Yin, S. Morgan, J. Yang, and Y. Zhang, "Practical compressive sensing with toeplitz and circulant matrices," in Visual Communications and Image Processing, vol. 7744 of Proceedings of SPIE, July 2010.
[4] O. Wu, L. Østergaard, R. M. Weisskoff, T. Benner, B. R. Rosen, and A. G. Sorensen, "Tracer arrival timing-insensitive technique for estimating flow in MR perfusion-weighted imaging using singular value decomposition with a block-circulant deconvolution matrix," Magnetic Resonance in Medicine, vol. 50, no. 1, pp. 164-174, 2003.

[5] P. J. Davis, Circulant Matrices, John Wiley \& Sons, New York, NY, USA, 1979.

[6] Z. L. Jiang and Z. X. Zhou, Circulant Matrices, Chengdu Technology University Publishing Company, Chengdu, China, 1999.

[7] D. Chillag, "Regular representations of semisimple algebras, separable field extensions, group characters, generalized circulants, and generalized cyclic codes," Linear Algebra and Its Applications, vol. 218, pp. 147-183, 1995.

[8] Z.-L. Jiang and Z.-B. Xu, "Efficient algorithm for finding the inverse and the group inverse of FLS $r$-circulant matrix," Journal of Applied Mathematics \& Computing, vol. 18, no. 1-2, pp. 45-57, 2005.

[9] J. Li, Z. Jiang, and N. Shen, "Explicit determinants of the Fibonacci RFPLR circulant and Lucas RFPLR circulant matrix," JP Journal of Algebra, Number Theory and Applications, vol. 28, no. 2, pp. 167-179, 2013.

[10] Z. L. Jiang, J. Li, and N. Shen, "On the explicit determinants of the RFPLR and RFPLL circulant matrices involving Pell numbers in information theory," in Proceedings of the International Conference on Information and Computer Applications (ICICA '12), vol. 308 of Communications in Computer and Information Science, pp. 364-370, 2012.

[11] Z. Tian, "Fast algorithms for solving the inverse problem of $A X=b$ in four different families of patterned matrices," Far East Journal of Applied Mathematics, vol. 52, no. 1, pp. 1-12, 2011.

[12] N. Shen, Z. L. Jiang, and J. Li, "On explicit determinants of the RFMLR and RLMFL circulant matrices involving certain famous numbers," WSEAS Transactions on Mathematics, vol. 12, pp. 42-53, 2013.

[13] D. V. Jaiswal, "On determinants involving generalized Fibonacci numbers," The Fibonacci Quarterly, vol. 7, pp. 319-330, 1969.

[14] D. Lin, "Fibonacci-Lucas quasi-cyclic matrices," The Fibonacci Quarterly, vol. 40, no. 3, pp. 280-286, 2002.

[15] D. A. Lind, "A Fibonacci circulant," The Fibonacci Quarterly, vol. 8, no. 5, pp. 449-455, 1970.

[16] S.-Q. Shen, J.-M. Cen, and Y. Hao, "On the determinants and inverses of circulant matrices with Fibonacci and Lucas numbers," Applied Mathematics and Computation, vol. 217, no. 23, pp. 9790-9797, 2011.

[17] M. Akbulak and D. Bozkurt, "On the norms of Toeplitz matrices involving Fibonacci and Lucas numbers," Hacettepe Journal of Mathematics and Statistics, vol. 37, no. 2, pp. 89-95, 2008.

[18] F. Yilmaz and D. Bozkurt, "Hessenberg matrices and the Pell and Perrin numbers," Journal of Number Theory, vol. 131, no. 8, pp. 1390-1396, 2011.

[19] J. Blümlein, D. J. Broadhurst, and J. A. M. Vermaseren, "The multiple zeta value data mine," Computer Physics Communications, vol. 181, no. 3, pp. 582-625, 2010.

[20] M. Janjić, “Determinants and recurrence sequences," Journal of Integer Sequences, vol. 15, no. 3, pp. 1-12, 2012.

[21] M. Elia, "Derived sequences, the Tribonacci recurrence and cubic forms," The Fibonacci Quarterly, vol. 39, no. 2, pp. 107-115, 2001. 


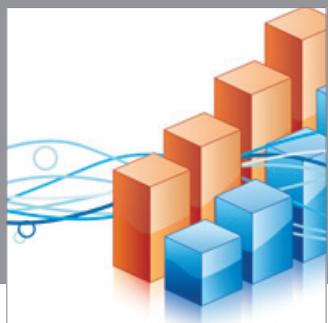

Advances in

Operations Research

mansans

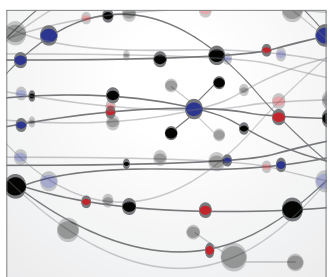

The Scientific World Journal
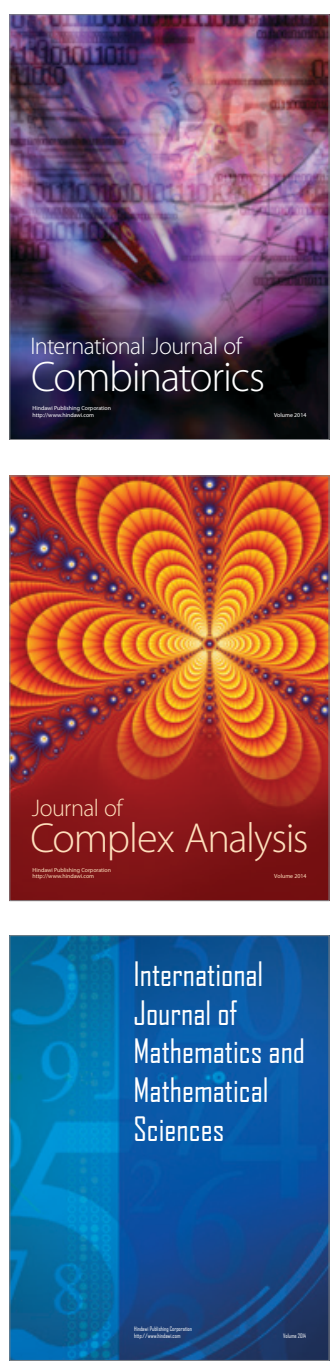
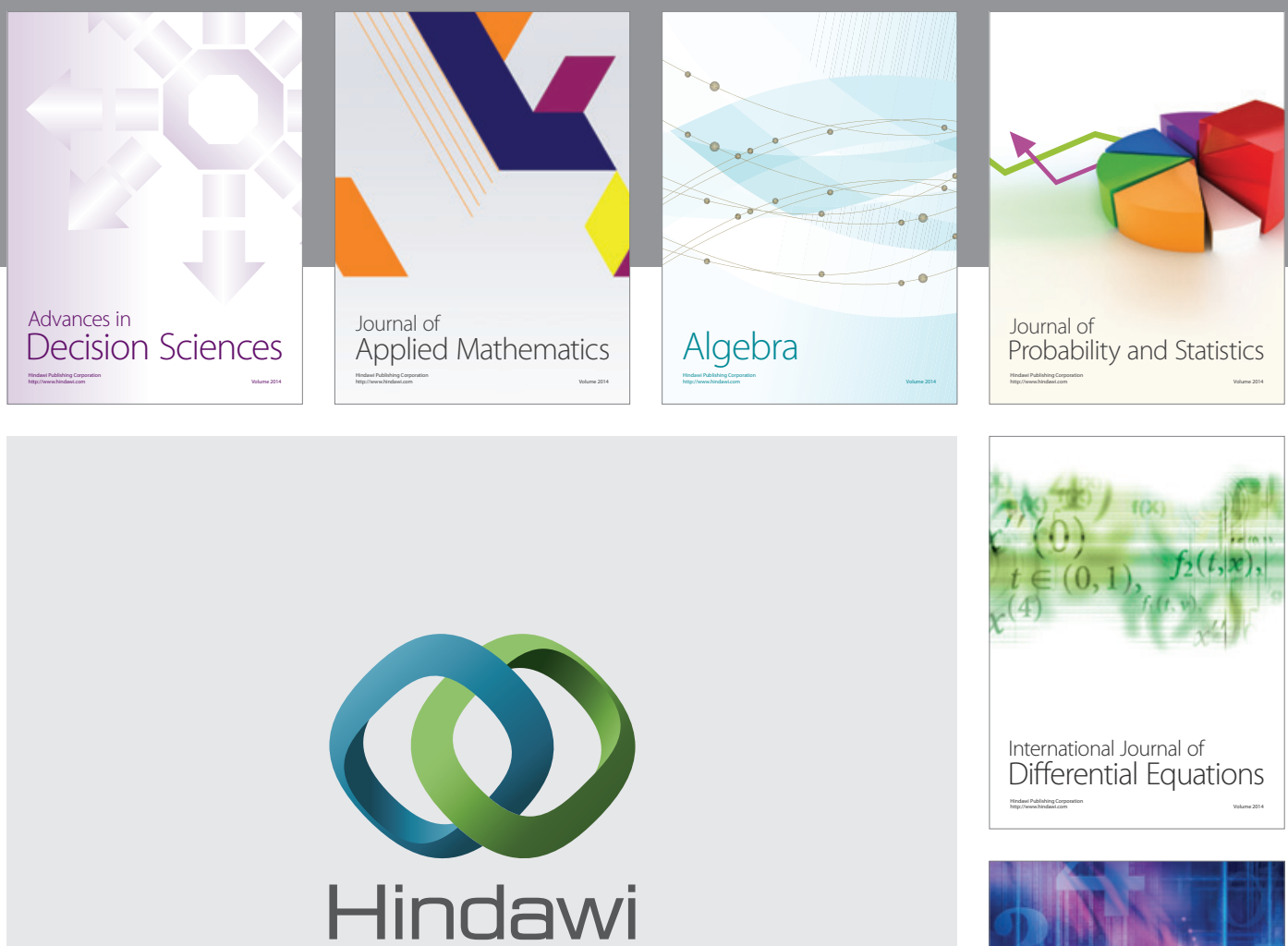

Submit your manuscripts at http://www.hindawi.com
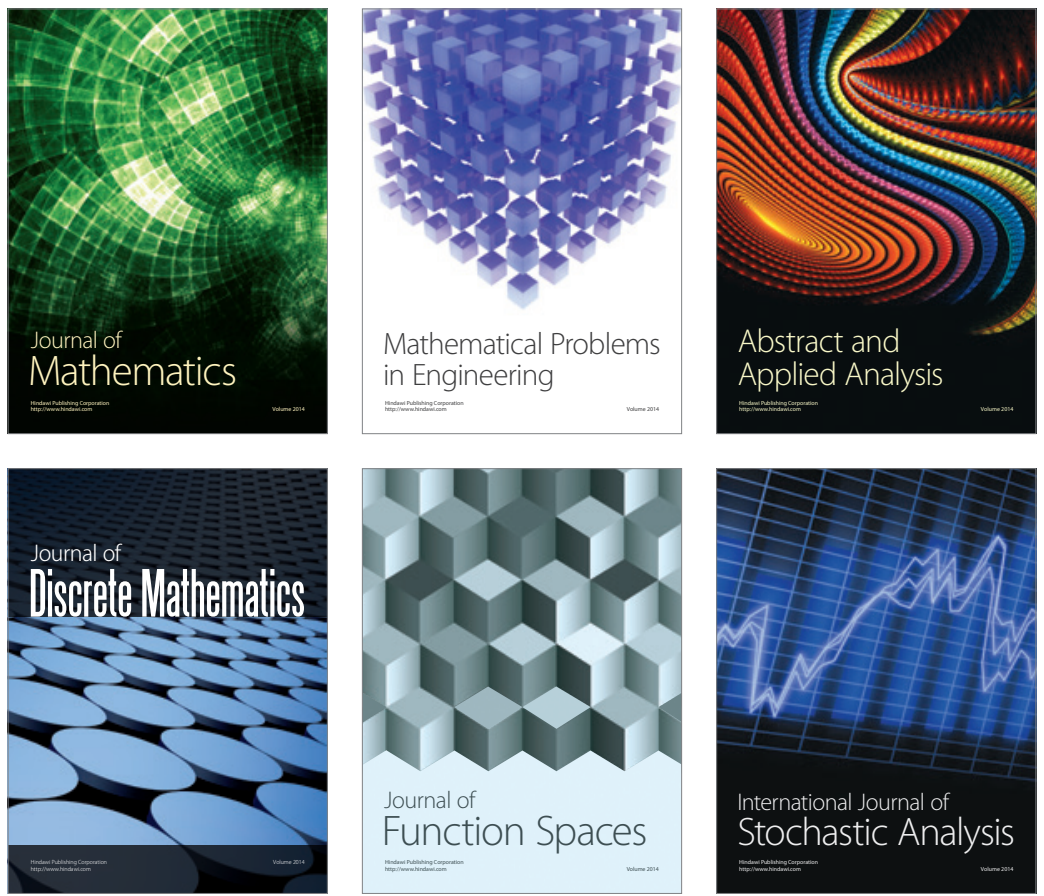

Journal of

Function Spaces

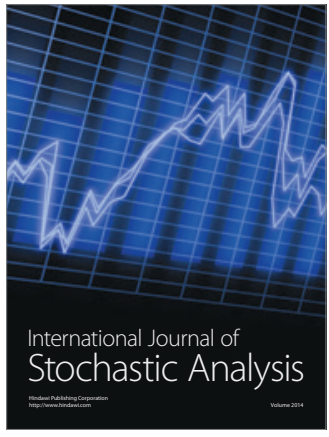

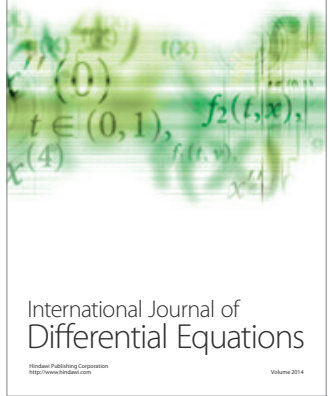
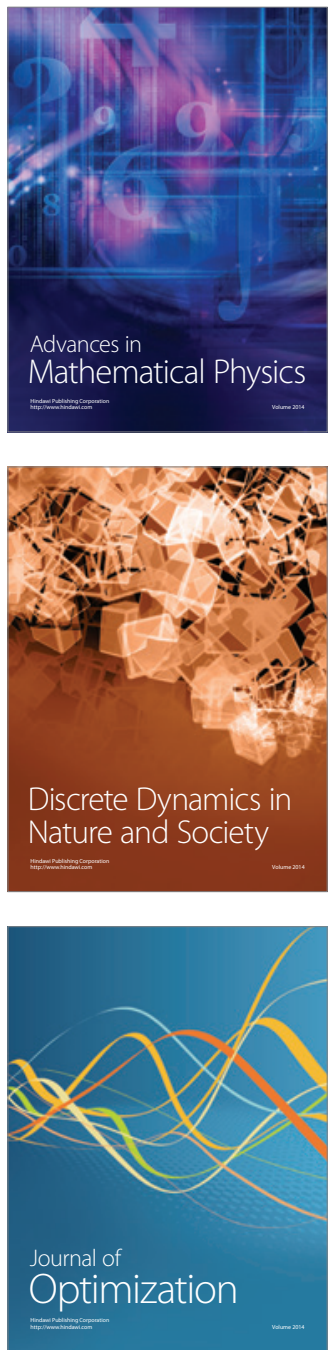